\title{
eCommons@AKU
}

January 2011

\section{A Qualitative Study: Why Prolonged Stay in the Recovery Room?}

Sharifa B. Lalani

Aga Khan University

Fauzia Ali

Aga Khan University

Zeenatkhanu Kanji

Aga Khan University, zeenatkhanu.kanji@aku.edu

Salma Jaffer

Aga Khan University

Mohammed Ali

Aga Khan University

Follow this and additional works at: http://ecommons.aku.edu/eastafrica_fhs_sonam

Part of the Nursing Commons

\section{Recommended Citation}

Lalani, S. B., Ali, F., Kanji, Z., Jaffer, S., Ali, M. (2011). A Qualitative Study: Why Prolonged Stay in the Recovery Room?. British Journal of Anaesthetic \& Recovery Nursing, 12(1-2), 9-13.

Available at: $\mathrm{http}: / /$ ecommons.aku.edu/eastafrica_fhs_sonam/64 


\title{
A Qualitative Study: Why Prolonged Stay in the Recovery Room?
}

\author{
Sharifa B. Lalani ${ }^{1 *}$, Fauzia $\mathrm{Ali}^{1}$, Zeenatkhanu Kanji ${ }^{1}$, Salma Jaffer ${ }^{2}$, Mohammed $\mathrm{Ali}^{3}$ \\ ${ }^{1}$ The Aga Khan University School of Nursing, Karachi, Pakistan; ${ }^{2}$ The Aga Khan University \\ Hospital, Karachi, Pakistan; ${ }^{3}$ Department of Anesthesiology, The Aga Khan University Hospital, \\ Karachi, Pakistan
}

\begin{abstract}
There are a number of factors that prolong patients' stay in the recovery room (RR), which are related to system or clinical issues. However, less has been discovered from the RR nurses about reasons for prolonged patient stay and recommendations to solve this issue. Prolonged patient stay of more than 2 hours in the RR interferes with the primary role of the RR nurse, which is to provide care to immediate postanaesthesia patients. Consequently, this could affect the operating room schedule, normal flow of patients to the RR and discharge to the nursing units.
\end{abstract}

Key words: Recovery room; Qualitative research; system and clinical

\section{INTRODUCTION}

The recovery room (RR) has evolved from a general to intensive care specialty, thereby creating the need to provide complex care to patients. Patient numbers and acuity is constantly changing in the RR, which results in delays in discharging patients to the nursing units, eventually developing bottlenecks within the RR [Smedley, 2010]. This creates additional demands on the RR nurses, because in many major surgeries the

Correspondence to: S. B. Lalani, RN, RM BScN, MScN, STTI Rho.Delta Chapter, Senior Instructor, The Aga Khan University School of Nursing, Stadium Road, PO Box 3500, Karachi 74800, Pakistan. E-mail: sharifa.lalani@aku.edu; Tel: +92 2134930051 Ext.: 5443, Direct: 3486 5432; Fax: +92 21493 4294/493 2095 patients' stay in the RR is of a longer duration, as they require careful and detailed clinical monitoring for safe recovery. The transfer of the patient from the RR to their designated unit is delayed because of: (a) unavailability of beds; (b) anaesthesia evaluation of patients; (c) floor nurses being too busy to receive patients; and (d) unavailability of porters to transfer the patients [Bruce, 2000]. Some reasons for the prolonged stay studied in Pakistan are: the patients' need for postoperative monitoring, unavailability of special care beds, pain management and postoperative ventilation [Samad et al, 2006]. However, none of the studies have considered exploring nurses' experiences, contributing to prolonged-stay patients in the RR.

\section{METHODS}

A qualitative descriptive-exploratory design guided this study. It is the method of choice as it gives the researcher a clear picture of an occurrence or a phenomenon [Sandelowski, 2000]. The ethics approval was granted from the Ethical Review Committee of the Aga Khan University. Semi-structured interviews were used to collect data that lasted from 45-60 minutes. Purposive sampling was utilised because participants are best able to answer the research questions and get a sense of the special experiences that they have undergone [Polit et al, 2001]. Hence, a group of six RR nurses who met the inclusion criteria were included (see Table 1). Confidentiality and anonymity were maintained by giving pseudonyms to the participants. The raw data were kept under lock and key. All the interviews were conducted in Urdu because of the participants' language preference, and probes were used 
Table 1. Inclusion and exclusion criteria

Full time registered nurses of both gender

Two or more than 2 years of experience as a bedside nurse and as a team leader

Caring for ventilated and non-ventilated prolonged-stay patients Willing to participate in the study

Willing to have their interview recorded either in Urdu or English Nurses on leave during the study period were excluded

to give structure and direction to the interview. Data analysis was also done as an activity, simultaneously with the data collection process, to handle the voluminous data. Moreover, the interviews were read through several times to get a deeper understanding of the narrative data. The content was organised and the narrative was integrated according to the emerging subcategories and categories [Polit and Beck, 2008].

Trustworthiness is the term used by a qualitative researcher to ensure the rigour of the study. Criteria of credibility, dependability, conformability and transferability were utilised [Lincoln and Guba, 1985]. Credibility was maintained by member check. Member checks were carried out formally and informally with the participants after the interviews, in order to verify that what was transcribed was what was intended to be communicated. Dependability was accomplished by being cautious at each step of the research process. Conformability was established by maintaining the audit trail through the record of raw data (interview transcript), data reduction notes (theoretical notes) and the emerging categories, subcategories and a theme. Transferability was achieved by documenting a thick description, which would allow readers to decide the extent to which the findings could be transferable.

\section{FINDINGS}

The overarching theme that emerged from the research questions was factors impacting quality of patient care. The qualitative analysis generated two broad categories: organisational factors and recommendations. The subcategories for organisational factors were system and clinical issues, whereas subcategories for recommendations were management and structural changes (see Table 2).

\section{Category 1: organisational factors}

Organisational factors were defined as the aspects related to the availability of the beds and the type of surgery.
Table 2. Categories and subcategories

\author{
Organisational factors \\ System issues \\ Clinical issues \\ Recommendations \\ Management changes \\ Structural changes
}

\section{Subcategory 1: system issues}

The system issues included shortage of beds, space constraint in the RR, anaesthetists' other responsibilities, unavailability of monitoring facilities in other units and VIP (very important person) patients.

Shortage of beds. Most of the participants identified shortage of beds, including general surgery beds, special care beds and intensive care beds in the hospital because of which patients' stay was prolonged in the RR. Saba shared that, 'The main challenge is the issue of patients' beds. When we want to transfer the patient to the special care unit, there is no bed vacant'.

Space constraints in the RR. There are a total of $14 \mathrm{RR}$ beds, out of which 1-2 beds are for ventilated patients and 3-4 beds are often occupied by overnight patients. Naila expressed that 'Most patients have a genuine need for staying in the RR and being closely monitored, but we have extremely limited space in the RR'.

Anaesthetists' other responsibilities. Most of the participants shared that when the RR anaesthetist is busy in other areas of the hospital, then patients' stay is prolonged in the RR. Saba said, 'One reason of delayed transfer of patient would be the unavailability of the anesthetist; an RR anesthetist is supposed to look after rush [emergency] calls, trauma calls, and ECT's [electro convulsive therapies], [therefore] shifting of patients is delayed'.

Unavailability of monitoring facilities in other units. Another reason for the prolonged stay was unavailability of non-invasive and invasive monitoring facilities in other units, for example, absence of arterial-line monitoring facilities in the surgical day care (SDC) unit, and noninvasive facilities in the magnetic resonance imaging for postanaesthesia patients. Kiran explained that 'Sometimes in SDC [surgical day care], the monitoring [equipment] is not available so if a patient gets sick he is accommodated in the main RR'.

VIP patients. Another reason shared by the participants was the accommodation of VIP patients in the RR. According to Saira's experience, 'Sometimes just because of their VIP status some patients have to be accommodated either overnight or even for a day or two'. In order to ensure vigilant monitoring, VIP patients stay for more than 
one night or until the nurses receive orders from their surgeons to transfer them to the designated unit.

\section{Subcategory 2: clinical issues}

Some of the reasons for prolonged-stay patients in the $\mathrm{RR}$ are medical reasons associated with the condition of the patient, such as ASA (American Society of Anesthesiologist) levels, type of surgery, type of anaesthesia and nurses' competency.

ASA level. Sometimes patients' stay is prolonged in the RR because of ASA levels. The higher the ASA level, the more crucial becomes the need for vigilant observation. According to Naila, 'The duration of stay depends on co-morbidities and ASA levels and if it is 3 or 4 , then definitely that patient needs to stay longer than the other patients'.

Type of surgery. A major surgery or any extensive surgery may also prolong the patient's stay in the RR. For example, Naila shared, 'Patients who undergo major surgical procedures like Whipple's procedure [it is done for pancreatic cancer] or hemicolectomy [removal of colon] require prolonged stay in the RR for crucial assessment and observation'.

Type of anaesthesia. The duration of stay in the RR also depends on the type of anaesthesia, like general anaesthesia patients take time in regaining consciousness, whereas in regional anaesthesia, a patient's restoration of lower limb movements is quite delayed. According to Nasim, 'Deep anesthesia patients take much longer to regain consciousness'.

Nurses' competency. Surgeons prefer specialty units because nurses are competent to take care of postoperative patients.

As Saira shared, 'In case of a urology patient, the doctor prefers to send the patient to the B1 ward because the doctors think that the nurses of $\mathrm{C} 1$ are not aware about catheter care following TURP [Transuretheral resection prostate] They fear that if it gets blocked, it would be harmful for the patient, so they encourage patients to stay in the RR until they get a bed in the B1 unit'.

\section{Category 2: recommendations}

Recommendations are related to the reorganisation of the RR and restructuring of the management of surgical patients in the hospital. There are two subcategories under this heading: 'management changes' and 'structural changes'.

\section{Subcategory 1: management changes}

In this subcategory, recommendations related to management changes, such as RR staffing, nurses competency, operating room (OR) schedule, narcotics dispensing, coordination among teams and RR policy have been shared by the participants.

$R R$ staffing. The participants emphasised the importance of adequate staffing. Naila's recommendation was, 'we should get a UR (unit receptionist)... whose job is to book beds of prolonged-stay patients, do paging, receive calls, call units to check the availability of beds etc. We shouldn't have to do all this'. Nasim shared that 'sometimes there is so much influx of patients that two nursing staff transfer the patients to their designated units, so more porters are required in the RR'.

Kiran shared another suggestion for better management: 'We don't have a bed coordinator [who arranges beds for the RR patients] availability after 4 o'clock...I think it would really help if a bed coordinator is assigned in the evening also'. In addition to this, she said, 'More staff should be assigned in the evening shift, or management staff [Head Nurse/Clinical Nurse Instructor] should do evenings, so that the TL [Team Leader] has fewer problems to face'. Tania suggested that to overcome the staffing issue, 'this shortage of nurses should be overcome by hiring more nurses from outside and good salary packages should be offered to retain them'.

Nurses competency. Most of the participants shared that the competency of all the nursing staff working in the units/wards should be such that they can manage all types of patients. Saba shared an example, 'C1 (medical/surgical ward) staff should be trained adequately to take care of the urology patient; so if there is an orthopedic patient, a B1 (surgical ward) staff should be trained enough to look after that orthopedic patient'.

OR schedule. One of the participants shared her views about the OR schedule that significantly impacts the RR patients, because overbooked OR schedule can overcrowd the RR as well. According to Kiran, 'Patient care is really important. Cases [electives] would be handled anyway, but first you need to deal with the emergency cases, so taking limited cases per day, would decrease the influx in the RR and improve the patient care'.

Narcotic dispensing. Two of the participants talked about indirect tasks like narcotic dispensing that takes them away from patient care. Nasim commented about narcotics dispensing, 'I pray to God that this responsibility would be taken away from the RR; this will enable us to give the time spent on dispensing narcotics to patients, who rightly deserve our consistent attention'.

Co-ordination amongst teams. Saba stressed the need for co-ordination and collaboration between the anesthesia and the primary team. She said, 'There should be coordination between anesthesia and the primary team, so that patient shifting is done timely and properly. 
There ... proper coordination between nurses and the primary team also... they say ... they are coming, but don't turn up'.

$R R$ policy. Tania recommended that there should be a strict policy of receiving ventilated patients in the RR; 'We don't have any policy in recovery, we just get a call that, okay, we are sending a patient to the RR, put him on the vent. There is no proper system and people just act according to their own wishes'.

\section{Subcategory 2: structural changes}

In this subcategory, recommendations related to structural changes, such as the expansion of the hospital beds including the RR, surgical intensive care unit (SICU) and the postoperative critical care unit (POCCU), will be shared through quotes.

Expansion of the RR. A few of the participants stated that the present space in the main RR is not adequate and that it should be reconstructed. Tania elaborated, 'The whole RR needs to be reconstructed. The number of monitors should be increased, plus the space of the RR should also expand, so that both patients and the staff benefit from the change'.

Saba recommended, 'Special care beds should be increased in hospital... main reason ... gets prolonged in the RR, number of ICU beds should be increased ... very sick patients come to hospital ... no space in the ICU, stay prolonged in RR'.

SICU and POCCU. A few of the participants identified the need for an SICU and a POCCU where major surgery patients should be cared for so that postoperative patients' quality of care is not compromised. Saira recommended, 'A surgical ICU (with separate staffing, an anesthetist and a surgery resident) should be set up where we can accommodate critical [patients] who are intubated and extubated'.

\section{DISCUSSION}

In the current study, the reasons related to patients' prolonged stay in the RR were: (a) shortage of beds; (b) the anaesthetist being busy in other units; (c) postoperative monitoring; (d) type of surgery; (e) type of anaesthesia given to the patient; (f) postoperative complications; and (g) increased ASA levels, whereas reasons for prolonged stay in a study done in Israel and Pakistan were: postoperative monitoring and mechanical ventilation [Samad et al, 2006; Ziser et al, 2002]. In addition, pain management and unavailability of special care beds were also identified as reasons in a Pakistani study done by Samad et al [2006]. In the current study, patients were received from the SDC OR for monitoring purposes.
The reason for this is that the SDC OR closes by evening and patients requiring observation are shifted to the main $\mathrm{RR}$ because it is equipped with invasive monitors and it is open 24 hours a day. In addition, competent nursing staff and an on-call anaesthetist are available in the RR to monitor and care for the patients.

The other finding in the current study was the prolonged stay of VIP patients in the RR. Medical management of VIP patients often poses unusual difficulties, as they need a lot of attention by the health-care professionals [Mariana and McLeod, 2007] In addition to this, the participants also identified that one reason for the prolonged stay of the patients in the RR is that doctors prefer admitting patients to specialty units. This is because the nurses in these units are competent in looking after the patients admitted to their units.

Several recommendations were suggested by the participants to overcome the above-mentioned challenges. As mentioned earlier, an anaesthetist and a surgical consultant should be designated to the RR for taking prompt decisions for the RR patients. These recommendations are consistent with the Samad et al [2006] and Ziser et al [2002] studies, which suggested that surgical consultation should be available at all times and a dedicated anaesthetist should be available for the RR patients. In the current study, participants recommended that the RR staffing should be increased, which includes nurses, unit receptionist, porters and bed co-ordinator. The participants further recommended that $\mathrm{OR}$ narcotics should be directly dispensed by the OR pharmacy so that the RR nurses could spend more time in direct patient care. This finding is similar to that of Merkouris et al [2004], who studied patient satisfaction in a Greek hospital and found that patients expected that more time should be spent on direct care delivery, as their needs remained unmet because of shortage of nurses.

A few participants in this study recommended that, for critical surgical patients, there should be a SICU rather than admitting them to the RR. Similarly, Jones and Harper's [2002] study also proposed setting up a POCCU, where only postoperative critical care patients are admitted and cared for. Participants in the current study highlighted that the RR should be renovated and beds should be increased. Literature recommends a ratio of 1.5-2 RR beds to $1 \mathrm{OR}$, which is necessary to provide safe care to the postoperative patients [Drain, 2003]. Moreover, the participants suggested that the OR schedule should be more organised so that the RR overcrowding could be avoided. In addition, the participants identified that at the time of admission, appropriate beds should be allocated to the patients and this suggestion was found consistent with the Ziser et al [2002] study. 


\section{LIMITATIONS}

In view of the economics of today's health care, one of the limitations of this study was that the nurses were unable to look at the entire situation comprehensively and globally. Nurses need to broaden their horizon by being productive, resourceful and accountable of providing a high quality of nursing care.

\section{CONCLUSION}

The findings of the study conclude that there are many reasons for patients' prolonged stay in the RR, and out of these, some are related to system or clinical issues. The organisational factors that are related to the system can be worked upon and the system can be improved, such as the unavailability of beds. However, some of the clinical issues, such as postoperative complications, are sometimes unavoidable. The POCCU can take care of all the patients requiring prolonged and complex care following surgery.

\section{STUDY OUTCOME}

The implementation of the study has been started as the research findings have been shared with the RR management. The separate four-bedded postoperative critical care area has been identified in the main $\mathrm{RR}$ for critical care patients requiring close observation and monitoring. The evening staffing has been increased because more patients are shifted to the RR from the OR during the evening. Some of the OR narcotics like Pethidine have been dispensed through the OR pharmacy. The unit receptionist has been employed to deal with indirect tasks so that nurses can spend more time on direct patient care. In addition to that, the morning shift bed co-ordinator arranges the special care beds and in the evenings, the nursing supervisor deals with the problem. Moreover, there is improved co-ordination and collaboration among the intensive care unit (ICU) and the RR team. Ventilated patients are transferred to the ICU as soon as a bed is available. The issue of the prolonged stay in the RR continues to be a complex problem but could be worked out through a multi-disciplinary team approach.

\section{ACKNOWLEDGEMENTS}

The author acknowledges all the study participants who have taken their precious time to share their experiences.

\section{REFERENCES}

Bruce M. A study in time: performance improvement to reduce excess holding time in recovery room. Journal of PeriAnesthesia Nursing 2000; 15(4): 237-241.

Drain CB. The Post Anesthesia Care Unit: A Critical Care Approach to Post Anesthesia Nursing. Philadelphia: W B Saunders, 2003.

Jones AG, Harper SJ. 'Ventilating in recovery' - the way forward: intensive therapy or postoperative critical care? British Journal of Anaesthesia 2002; 88(4): 473-474.

Lincoln YS, Guba EG. Naturalistic Inquiry. Newbury Park, CA: Sage, 1985.

Mariana EC, McLeod JA. Emergency care for the VIP patient. Intensive Care Medicine 2007; 20: 969-975.
Merkouris A, Papathanassoglou EDE, Lemonidou C. Evaluation of patient satisfaction with nursing care: quantitative or qualitative approach? International Journal of Nursing Studies 2004; 41: 355-367.

Polit DF, Beck CT. Nursing Research: Generating and Assessing Evidence for Nursing Practice. Philadelphia: Lippincott Williams \& Wilkins, 2008.

Polit DF, Beck CT, Hungler BP. Essential of Nursing Research: Methods, Appraisal and Utilization. Philadelphia: Lippincott, 2001.

Samad K, Khan M, Hameedullah, Fauzia AK, Hamid M, Fazal HK. Unplanned prolonged postanesthesia care unit length of stay and factors affecting it. Journal of Pakistan Medical Association 2006; 56(3): 108-112.

Sandelowski M. Whatever happened to qualitative description? Research in Nursing and Health 2000; 23: 334-340.

Smedley P. Safe staffing in the Post Anaesthetic Care Unit: no magic formula. British Journal of Anaesthetic \& Recovery Nursing 2010; 11(1): 3-8.

Ziser A, Alkobi M, Markovits R, Rozenberg B. The postanesthesia care unit as a temporary admission location due to intensive care and ward overflow. British Journal of Anaesthesia 2002; 88(4): 577-579. 